\title{
Contact-free calibration of an asymmetric multi-layer interferometer for the surface force balance
}

Marco Balabajew, Christian D. van Engers, and Susan Perkin

Citation: Review of Scientific Instruments 88, 123903 (2017);

View online: https://doi.org/10.1063/1.5006056

View Table of Contents: http://aip.scitation.org/toc/rsi/88/12

Published by the American Institute of Physics

\section{Articles you may be interested in}

High speed displacement measurement based on electro-magnetic induction applied to electromagnetically driven ring expansion

Review of Scientific Instruments 88, 114702 (2017); 10.1063/1.4989916

Invited Review Article: Measurements of the Newtonian constant of gravitation, G

Review of Scientific Instruments 88, 111101 (2017); 10.1063/1.4994619

Characterization of plastic and boron carbide additive manufactured neutron collimators

Review of Scientific Instruments 88, 123102 (2017); 10.1063/1.4998930

Effects of transients in LIGO suspensions on searches for gravitational waves

Review of Scientific Instruments 88, 124501 (2017); 10.1063/1.5000264

Optically transparent solid electrodes for precision Penning traps

Review of Scientific Instruments 88, 123101 (2017); 10.1063/1.5002180

Fitting a defect non-linear model with or without prior, distinguishing nuclear reaction products as an example

Review of Scientific Instruments 88, 115114 (2017); 10.1063/1.4993697

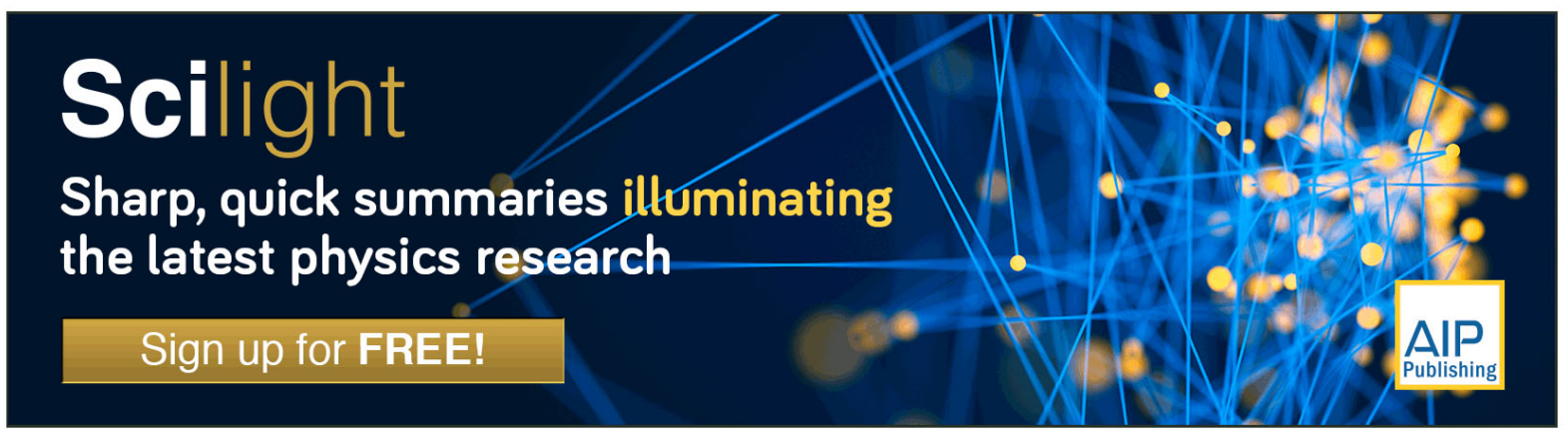




\title{
Contact-free calibration of an asymmetric multi-layer interferometer for the surface force balance
}

\author{
Marco Balabajew, ${ }^{\text {a) }}$ Christian D. van Engers, ${ }^{\text {a) }}$ and Susan Perkin ${ }^{\text {b) }}$ \\ Physical and Theoretical Chemistry Laboratory, Chemistry Department, University of Oxford, South Parks Road, \\ Oxford OX1 3QZ, United Kingdom
}

(Received 21 September 2017; accepted 16 November 2017; published online 8 December 2017)

\begin{abstract}
The Surface Force Balance (SFB, also known as Surface Force Apparatus, SFA) has provided important insights into many phenomena within the field of colloid and interface science. The technique relies on using white light interferometry to measure the distance between surfaces with sub-nanometer resolution. Up until now, the determination of the distance between the surfaces required a so-called "contact calibration," an invasive procedure during which the surfaces are brought into mechanical contact. This requirement for a contact calibration limits the range of experimental systems that can be investigated with SFB, for example, it precludes experiments with substrates that would be irreversibly modified or damaged by mechanical contact. Here we present a non-invasive method to measure absolute distances without performing a contact calibration. The method can be used for both "symmetric" and "asymmetric" systems. We foresee many applications for this general approach including, most immediately, experiments using single layer graphene electrodes in the SFB which may be damaged when brought into mechanical contact. Published by AIP Publishing. https://doi.org/10.1063/1.5006056
\end{abstract}

\section{INTRODUCTION}

The Surface Force Balance (SFB, also known as Surface Force Apparatus, SFA) has been used to provide important quantitative measurements of colloidal and surface forces across liquids, such as those arising due to van der Waals forces, ${ }^{1}$ structural forces, ${ }^{2}$ electrostatic surface forces, ${ }^{3-5}$ and hydration forces. ${ }^{6,7}$ The strength of the technique lies in the use of multiple beam interferometry to determine the separation between atomically smooth sheets of muscovite mica, with sub-nanometer precision. ${ }^{8}$ For this purpose, mirrors are applied to the backside of the mica sheets, forming an interferometer. The conventional approach to determine the distance between the two surfaces involves a contact calibration: the two surfaces are brought into mechanical contact prior to the start of the experiment, at which point the sum of the thicknesses of the two mica sheets can be determined. In the case of a symmetric system, i.e., involving two mica sheets of equal thickness, the distance between the surfaces can then be determined by means of analytical equations derived by Israelachvili. $^{8}$

In this paper, we present a general approach allowing for determining the thicknesses of the mica sheets or other spacers (see below) without performing a contact calibration. The method can be used for both symmetric systems-in which the thickness of the spacer layers is equal-and asymmetric systems. First, we will show how secondary fringe patterns can be used to obtain suitable initial guesses for the spacer thicknesses. We will then show how these initial guesses can be used in combination with their characteristic periodicity to provide input parameters for a brute-force search

\footnotetext{
a) M. Balabajew and C. D. van Engers contributed equally to this work.

b) Author to whom correspondence should be addressed: susan.perkin@ chem.ox.ac.uk
}

based on the primary fringe patterns, allowing us to obtain the spacer layer thickness on a desktop computer within a day, rather than weeks - the time required to perform this calculation without using this method. Finally, we demonstrate the strength of this approach by measuring structural forces in the ionic liquid 1-ethyl-3-methylimidazolium bis (trifluoromethylsulfonyl)imide $\left(\left[\mathrm{C}_{2} \mathrm{C}_{1} \operatorname{Im}\right]\left[\mathrm{NTf}_{2}\right]\right)$, for which we find excellent agreement with the force magnitude and liquid structural forces in the literature.

The optical elements that form the interferometer within the standard SFB-setup are shown in Fig. 1: back-silvered mica sheets of thicknesses $Z 1$ and $Z 2$ are glued mica-side up onto cylindrical quartz discs. We distinguish two distinct setups: symmetric, i.e., $Z 1=Z 2=Z$, and asymmetric, i.e., $Z 1 \neq Z 2$. The sheets are brought together in cross-cylinder configuration such that their separation $(D)$ is much smaller than their radius of curvature $(R)$ (i.e., $D \ll R$ ). In this configuration, white light passes through the interferometer, perpendicular to both surfaces, and produces multiple sets of interference fringes of equal chromatic order (FECO).

The primary fringes $P(D)$, which are used to measure the distance between the surfaces, arise due to reflections at the silver mirrors. Reflections between the silverlmica and micalmedium interfaces of a single mica sheet give rise to two sets of distance independent secondary fringes $S 1$ and $S 2$ (for symmetric setup, $S 1=S 2=S$ ). Additionally, two sets of tertiary fringes $T 1(D)$ and $T 2(D)$ arise due to the reflections at the silverlmica interface of one mica sheet and the micalmedium interface of the other mica sheet. These tertiary fringes are usually not observed due to their low intensities. Mica (coated with a silver mirror) has been employed as a substrate in the majority of SFB experiments to date due to its atomically smooth cleavage plane, high crystallinity and wellcharacterized optical properties. However, since the setup is not restricted to the use of mica, we will henceforth discuss 
(a)

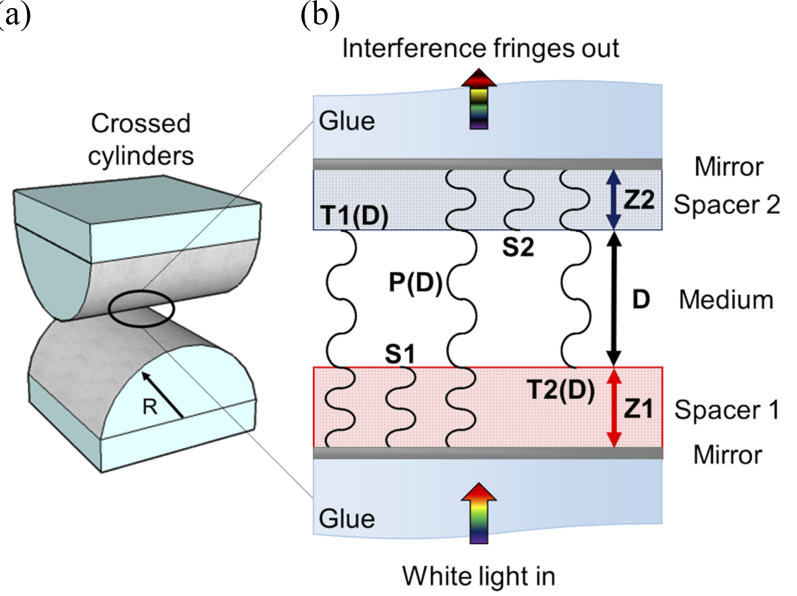

FIG. 1. Schematics showing (a) the crossed cylinder SFB-setup. In a typical experiment, the radius of curvature $\mathrm{R}$ is much larger than the distance between the surfaces $\boldsymbol{D}(\boldsymbol{R}>>\boldsymbol{D})$. (b) The interferometer inside the SFB: white light enters from the bottom and is partially reflected due to the mismatch between the refractive indexes of the different layers. Light that constructively interferes emerges at the other end. Primary fringes $[\boldsymbol{P}(\boldsymbol{D})]$ result from reflections between the mirrors. Secondary fringes $(\boldsymbol{S 1}, \boldsymbol{S 2})$ result from reflections between the mirror and the closest spacerlmedium interface. Tertiary fringes $[\boldsymbol{T 1}(D), T 2(D)]$ result from reflections between the mirror and the opposing spacerlmedium interface.

the interferometry using the generalized terms "spacer" and "mirror" instead of "mica" and "silver" (as in Fig. 1).

To determine the thickness of the two spacers $Z 1+Z 2$ in a regular SFB experiment, the spacers are first brought into contact, i.e., $D=0$, the so-called contact calibration. After contact calibration, the surfaces can be separated, and for $Z 1=Z 2=Z$ and $D \ll R$, changes in surface separation close to contact may be determined using the equations put forward by Israelachvili. ${ }^{8}$ Alternatively, $Z 1+Z 2$ may be determined numerically by matching the peaks in the transmitted interference spectrum in contact with a spectrum calculated via the multilayer matrix method. ${ }^{9-12}$ The latter treats the transmitted spectrum as the matrix product of the individual layers $\boldsymbol{M}$, each with its own characteristic matrix $\boldsymbol{M}_{\boldsymbol{i}}$ so that

$$
M=\prod_{i}^{N} M_{i},
$$

where

$$
M_{i}=\left[\begin{array}{cc}
\cos (q) & -\frac{i}{p} \sin (q) \\
-i p \sin (q) & \cos (q)
\end{array}\right]
$$

with

$$
q=\frac{2 \pi \tilde{n} z}{\lambda} \cos (\theta) \quad p=\sqrt{\frac{\varepsilon}{\mu}} \cos (\theta) .
$$

Here $\tilde{n}$ is the complex refractive index, $z$ is the layer thickness, $\lambda$ is the wavelength, $\theta$ is the angle between the incoming beam and the surface normal (for crossed cylinders with $D \ll R$, $\theta=0^{\circ}$ ), and $\varepsilon$ and $\mu$ are the dielectric constant (permittivity) and magnetic permeability, respectively. As such, for nonmagnetic materials, $\mathrm{p}$ effectively corresponds to the refractive index. We note that $\varepsilon$ and $\mu$ should not be multiplied by the permittivity of free space or the vacuum permittivity as was mistakenly suggested by Clarkson. ${ }^{12}$ The resulting transmission, $T$, through an interferometric stack containing $n$ individual layers has the form

$$
T=\frac{p_{0}}{p_{n+1}}\left|\frac{2 p_{0}}{\left(m_{11}+m_{12} p_{n+1}\right) p_{0}+\left(m_{21}+m_{22} p_{n+1}\right)}\right|^{2},
$$

where $m_{j k}$ refers to the element with the index $j, k$ of matrix $M$ and $p_{0}$ and $p_{n+1}$ are the values of $p$ for the semi-infinite adjacent layers. We will use the notation \{semi-infinite layer I layer 1 । layer $2 \ldots$ | layer $\mathrm{n} \mid$ semi-infinite layer $\}$ when referring to the interferometer stack.

Numerical determination of $Z 1$ and $Z 2$ using a contact calibration in a symmetric setup requires fitting to a 1 dimensional parameter space $\Xi=N^{1}$, using the stack $\{$ glue $\mid$ mirror $\mid$ spacer 1 | spacer 2 | mirror $\mid$ glue $\}$ since $Z 1=Z 2$. In the case of asymmetric spacer thicknesses, the parameter space increases roughly to $\Xi \approx 2 N^{1}$ by introducing a parameter that governs the asymmetry between $Z 1$ and $Z 2 .{ }^{11}$ More complex geometries may also be analyzed using a contact calibration. ${ }^{13}$

However, bringing the two surfaces into contact prior to performing the experiment is not always feasible, ${ }^{27}$ for example, when using sensitive surface coatings that may be irreversibly modified or damaged whilst going in/out of contact. ${ }^{14}$ As a result, the parameter space is enormously increased (to $\Xi=N^{2}$ for the symmetrical case and $\Xi=N^{3}$ for the asymmetrical case, \{glue I mirror I spacer1 I medium | spacer2 | mirror I glue \}). In this situation, numerically determining $Z 1$ and $Z 2$ becomes computationally unfeasible. In the case of asymmetric spacer thicknesses, we estimate the computation time to be multiple weeks on a desktop computer.

We have developed a method to measure the thicknesses of both surfaces, without performing a contact calibration. By analyzing the secondary fringes, which arise from reflections between the mirror and spacerlmedium interface ( $S 1$ and $S 2$ in Fig. 1), we obtain appropriate initial guesses for $Z 1$ and $Z 2$, which greatly speeds up the numerical calculation of the layer thicknesses.

In order to clarify this approach, we begin by considering the origin of the different FECO patterns observed. As discussed by Heuberger, ${ }^{15}$ primary fringes result from reflections between the mirrors and depend on the distance $D$ between them $[P(D)$ in Fig. 1]. Higher order fringes arise from reflections between the mirror and the spacerlmedium interface. The two sets of secondary fringes, $S 1$ and $S 2$, depend only on the thickness of their respective spacer thicknesses $Z 1$ and $Z 2$ [i.e., $S 1=S 1(Z 1)$ and $S 2=S 2(Z 2)$; see Fig. 1]. In the special case of a symmetric setup, there is only set of secondary fringes. Tertiary fringes depend both on $Z 1 / Z 2$ and on $D[T 1(D), T 2(D)$ in Fig. 1].

Typically (e.g., when using mica), the reflectivity of the mirror is much higher than that at the spacerlmedium interface. The relative intensity of the different fringe orders may be approximated using Snell's law giving $I_{P}>>I_{S}>I_{T}$. The intensity of each FECO pattern is divided over all fringe orders and depends on $D$ for $P(D)$ and $T 1(D), T 2(D)$. Therefore, when the separation between the surfaces is small, $P(D)$ dominates the FECO (Fig. 2, step 3). When the separation is large, $S 1$ and $S 2$ dominate (Fig. 2, step 1) and may be used to estimate $Z 1$ and $Z 2$. We note that it is important to match the refractive index 


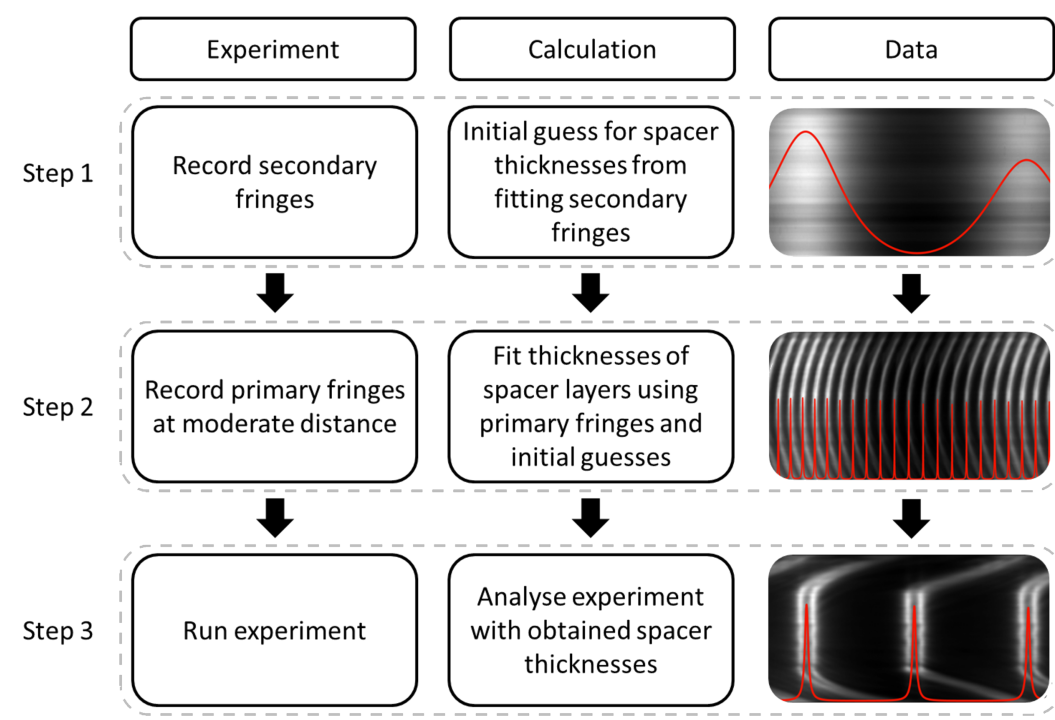

FIG. 2. Flow diagram demonstrating the steps involved in the contact free calibration method. Step 1: the secondary fringe traces are recorded at large surface separation while moving the surfaces and fitted to calculated spectra. Step 2: Primary fringes are recorded at moderate surface separation. The values obtained in step 1 are used as input parameters for a brute-force search to match the experimental primary fringes with calculated spectra. Step 3: The data collected during the experiment can be analyzed, i.e., surface separation $\boldsymbol{D}$ calculated for approaching surfaces, using the spacer thicknesses found in step 2 and without the need for a contact calibration. of the underlying glue to that of the underlying quartz disc (or vice versa), to prevent additional fringes resulting from reflections between the mirror and gluelquartz interface (outside of the intended interferometer). This is particularly important for asymmetric systems where the intensity of $S 1$ and $S 2$ is low (see Fig. S5 of the supplementary material).

Taking these principles into account and using a threestep procedure, we show that the method is robust for both symmetric and asymmetric interferometer setups. Finally, we present a proof-of-principle demonstration of the method.

\section{METHODS AND MATERIALS}

\section{A. Experimental setup}

Experiments were performed using a surface force balance as described by Kumacheva and Klein. ${ }^{16}$ A chargecoupled device (CCD; Retiga R6, QImaging) was used to record the interference fringes. The conversion factor to calculate wavelengths from pixel positions was obtained by measuring the positions of mercury emission lines. Muscovite mica (Ruby Mica Grade \#1 V-1/V-2, S\&J Trading, Inc.) was cleaved to obtain mica sheets corresponding to a single facet of $2.5-7.0 \mu \mathrm{m}$ in thickness and placed on a freshly cleaved thick mica plate as support and back-silvered (Silver shot, 99.9999\%, Alfa Aesar) using an HHV Auto306 (HVV Ltd.) thermal evaporator.

SFB lenses were prepared by gluing the mica sheets silver side down onto hemi-cylindrical quartz lenses using paraffin wax (melting point $=53-57{ }^{\circ} \mathrm{C}$ ASTM D87, Aldrich Chemistry). Gluing at $70{ }^{\circ} \mathrm{C}$ with as little paraffin as possible and slowly cooling to the freezemelt transition was necessary due to the slight contraction of the paraffin upon solidification. Alternatively, EPON 1002F epoxy resin could be used in combination with BaLF4 glass lenses. ${ }^{13}$ 1-ethyl-3-methylimidazolium bis(trifluoromethylsulfonyl)imide $\left(\left[\mathrm{C}_{2} \mathrm{C}_{1} \mathrm{Im}\right]\left[\mathrm{NTf}_{2}\right]\right)(99 \%)$ was obtained from IOLITEC ionic liquids technologies $\mathrm{GmbH}$ and dried under moderate vacuum $\left(5 \times 10^{-3}\right.$ mbar $)$ at $80{ }^{\circ} \mathrm{C}$ overnight.

\section{B. Experimental procedure}

Two pairs of lenses, each set consisting of mica sheets of equal thickness, were prepared. Both sets were separately mounted into the SFB and their fringes recorded. In this symmetric configuration where the mica sheets are of equal thickness $S 1=S 2$. It was pointed out by Heuberger ${ }^{15}$ that recording an image whilst moving one surface to vary $D$ may be used to average out any contribution of the primary fringes and obtain an image that shows only secondary fringes. Following this procedure, secondary fringes were recorded at large $D$ whilst moving the surfaces using a long exposure time for the CCD camera. Subsequently, the surfaces were brought into contact and the primary fringes $P(D)$ were recorded.

Next, one lens of each set was used to form an asymmetric configuration. For the asymmetric set, secondary and primary contact fringes were recorded again. In addition, primary fringes were recorded at a moderate distance, having $\sim 20$ fringes between the mercury reference lines.

In an independent experiment performed according to the same procedure, a set of lenses with mica sheets $(Z 1 \neq Z 2)$ was prepared. After recording secondary fringes and primary fringes at a moderate distance, $\left[\mathrm{C}_{2} \mathrm{C}_{1} \mathrm{Im}\right]\left[\mathrm{NTf}_{2}\right]$ was injected in between the surfaces. Then, the surfaces were slowly brought into close contact to measure the structural forces arising from the squeeze out of anion/cation layers in close confinement. ${ }^{17}$ Experiments were performed in $\mathrm{N}_{2}$ atmosphere. During the experiment, a small cup of $\mathrm{P}_{2} \mathrm{O}_{5}$ was placed in the chamber as desiccant.

\section{Data analysis and fitting}

For fitting the positions of the primary fringes, we used a method introduced by Heuberger. ${ }^{11}$ This technique uses the sum of intensities at the measured peak positions, calculated by the multilayer matrix method as a correlation function, to describe the similarity between the measured and calculated interference patterns and is chosen to minimize calculation time. This correlation function exhibits many local maxima at 
different parameter combinations. ${ }^{11}$ The maxima of this correlation function are equivalent to the local minima of sum squared residuals in conventional fitting approaches and prevent the optimal solution to be reliably found by conventional fitting algorithms. We therefore used a brute-force search to find the optimal fit.

For fitting the secondary fringe profiles, we used a conventional sum of squared residuals as a correlation function in combination with a brute-force search to find the optimal fit. The procedure to calculate the theoretical secondary profiles is described in detail in Sec. III. Optical constants for Ag and mica were taken from McPeak ${ }^{18}$ and Kienle. ${ }^{19}$

All computations were performed on a desktop computer (OptiPlex 7040, Dell) with an Intel Core i5-6500 CPU (3.2 GHz), 16 GB RAM and Windows 7 Enterprise 64-bit. The fitting software was home-written utilizing Armadillo ${ }^{20}$ as library for linear algebra.

\section{DATA ANALYSIS}

The general workflow of the data analysis is illustrated in Fig. 2. It consists of three major steps: (1) Initial guesses for the spacer thicknesses are obtained from the secondary fringe profiles. (2) The initial guesses are refined by fitting primary fringes measured at a moderate distance. (3) The obtained spacer thicknesses are used to evaluate the performed experiment. These steps will now be described in further detail.

\section{A. Step 1: Extracting secondary fringe information}

As explained in the Introduction, secondary fringes are a measure for the thicknesses of the spacer layers. In this section, we explain how to obtain good initial guesses for the thicknesses $Z 1$ and $Z 2$ (step 1 in Fig. 2).

Averaging over multiple distances greatly enhances the ability to extract secondary fringes $S 1$ and $S 2$ from the interferometric data. This is immediately clear when inspecting the large distance calculated spectrum (calculated for $\{$ glue $|\mathrm{Ag}|$ mica I air I mica I Ag I glue \}) shown in Fig. 3(a) (black dots). Here, the secondary fringes appear as an elevated background underneath the primary fringes [note that these are only visible due to adding an artificial offset to the refractive index of the Ag mirror of $\left.\left(n_{A g_{-} f i g 3 A}=n_{A g}+0.5\right)\right]$.

By calculating the same spectrum for a multitude of distances and averaging the result-analogous to averaging the CCD images with varying $D$ during the experiment-we remove the influence of the primary fringes and obtain the secondary fringe spectrum [Fig. 3(a), red solid line]. However, this calculation still involves multiple multilayer matrix calculations of $\{$ glue | mirror I spacer I medium I spacer I mirror I glue $\}$ to obtain a single profile and is therefore too computationally expensive for fitting the corresponding thickness $(Z 1, Z 2)$ to the experimentally obtained secondary fringe image. To circumvent this, we have found that a single 2-layer multilayer matrix calculation using $\{$ glue $\mid$ mirror $\mid$ spacer $\mid$ medium $\}$ is sufficient to calculate the secondary fringes [Fig. 3(a), blue line]. Here, the difference between the two calculated spectra (calculated for mica spacers, Ag mirrors, and air as medium) originates from the finite absorption in the mirror. (a)

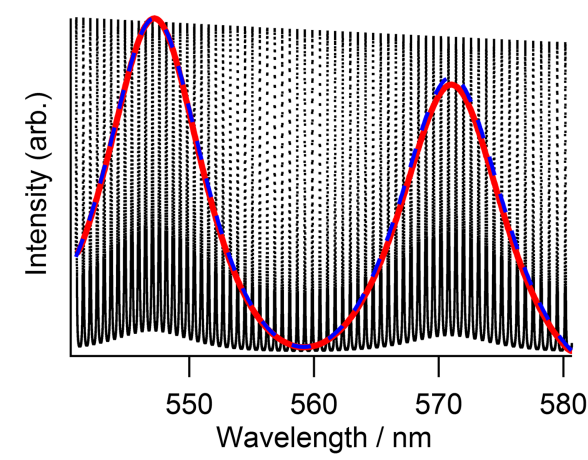

(b)

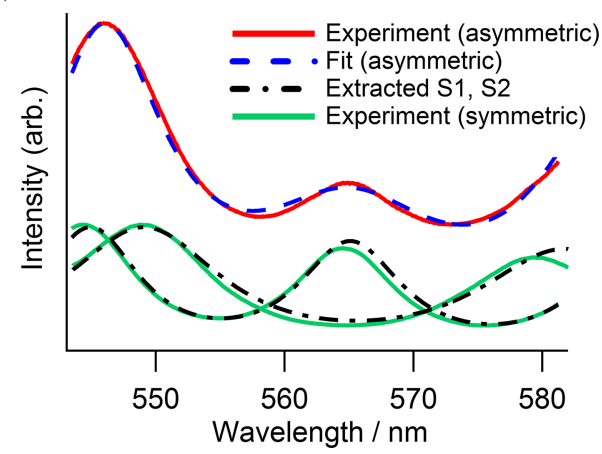

FIG. 3. Extracting thicknesses of the spacer layers from secondary fringe patterns. (a) Comparison of methods to calculate secondary fringe patterns: The black dots show the secondary fringes present as an offset (two humps) underneath the primary fringes (calculated for $\{$ glue $|\mathrm{Ag}|$ mica I air I mica $|\mathrm{Ag}|$ glue $\}$ ). The red line shows the results of averaging multiple of these calculations, at different air thicknesses $\boldsymbol{D}$, effectively removing the influence of the primary fringes. The blue dashed line is a calculation using \{glue $|\mathrm{Ag}|$ mica $\mid$ air $\}$. (b) Comparison of experimental data and fits: The red line shows the secondary fringe trace obtained in asymmetric configuration. The fit to the data is shown in blue dashed line and was obtained by summing calculated intensities $\boldsymbol{I}_{\boldsymbol{S 1}}$ and $\boldsymbol{I}_{\boldsymbol{S 2}}$ using Eq. (5). The dashed-dotted lines show how the values $\boldsymbol{I}_{\boldsymbol{S 1} \mathbf{1}}$ and $\boldsymbol{I}_{\boldsymbol{S} \mathbf{2}}$ from the fit compare to secondary fringe patterns obtained experimentally in symmetric configuration (green solid line).

To fit the total intensity $I_{S 1+S 2}$ for the asymmetric configuration, using the intensity of the secondary fringe profiles for $S 1$ and $S 2\left(I_{S 1}, I_{S 2}\right)$ as input parameters, we attempted different summing procedures. We found that

$$
I_{S 1+S 2}=\frac{I_{S 1} I_{S 2}}{I_{S 1}+I_{S 2}-I_{S 1} I_{S 2}}
$$

provides closest agreement with experimental data, compared to using summation, the reciprocal sum, or Airy's formulae ${ }^{9}$ (see the supplementary material). This summing procedure for secondary fringes is further called "sum."

Figure 3(b) shows the results obtained through this procedure for the asymmetric configuration of a stack of \{glue | Ag | mica $\mid$ air $\mid$ mica $|\mathrm{Ag}|$ glue $\}$. First, the experimental secondary fringe profile (red solid line) was extracted and a sum [according to Eq. (5)] of two \{glue | Ag I mica | air $\}$ calculations, with thicknesses $Z 1$ and $Z 2$ as fitting parameters, was fitted to the experimental profile. The fit (blue dashed line) shows good agreement with the experimental data and provides good starting parameters for further refinement.

For comparison, the fringe profiles for the fitted thicknesses $Z 1$ and $Z 2$ are shown (black dashed-dotted line) next 
to their experimental counterpart (green solid line), measured in symmetrical configuration. The fits show excellent agreement with fits obtained for the symmetrical configurations and deviate only by $3.7 \mathrm{~nm}$ for one fringe profile and by $171.2 \mathrm{~nm}$ for the other. The larger difference of the latter corresponds to the distance between two consecutive fringe orders, which satisfies the condition

$$
d_{\text {fringe orders }}(\lambda)=\frac{\lambda}{2 n_{\text {spacer }}} .
$$

For the wavelength range of interest here, this equates to $\mathrm{d}_{\text {fringe orders }}(540 \mathrm{~nm}) \approx 169 \mathrm{~nm}$ and $\mathrm{d}_{\text {fringe orders }}(585 \mathrm{~nm})$ $\approx 183 \mathrm{~nm}$.

We have performed multiple experiments where we fit the asymmetric secondary fringe profiles and compare the values for $Z 1$ and $Z 2$ with those fitted to secondary fringes obtained in the symmetrical configurations. We always find agreement either within a few nanometers or within a few nanometers plus/minus one fringe order. This clearly shows the strength of this approach in predicting the spacer thicknesses. Overall, these steps result in a sufficiently good estimate of $Z 1$ and $Z 2$ to proceed to the calculation of $D$ and refinement of $Z 1$ and Z2 using primary fringe information.

\section{B. Step 2: Refining spacer thicknesses using primary fringe information}

The spacer thicknesses obtained in the first step are only an initial guess for the actual thicknesses. In the following paragraphs we describe how to refine the obtained thicknesses $Z 1$ and $Z 2$ to get an accurate absolute distance when evaluating the data obtained during the experiment (step 2 in Fig. 2).

As shown before, $Z 1$ and $Z 2$ obtained by a fit of a secondary fringe profile for an asymmetric configuration can be shifted by one fringe order (approximately $175 \mathrm{~nm}$ in the wavelength range used for the shown data). To get more accurate values for $Z 1$ and $Z 2$, we measure a primary fringe pattern in air at a moderate distance (approximately 20 primary fringes should be in view which in our setup corresponds to a distance on the order of $50 \mu \mathrm{m}$ ). The evaluation of the fringe positions involves multiple steps. First, a rough estimate for the gap thickness is needed. To get this initial guess, the measured primary fringe positions are obtained by fixing $Z 1$ and $Z 2$ to the initial guesses (see step 1) and fitting positions of the primary fringes with the gap thickness as fitting parameters. It should be noted that this results only in a rough estimate of $D$. The range of $D$ used in the parameter space for the final fitting of primary fringe positions should still be large. We recommend using a range of $\pm 5 \%$ around the initial guess for this parameter.

For the final fitting procedure, a suitable parameter range for $Z 1$ and $Z 2$ needs to be chosen as well. The parameter space should be chosen as small as possible since it directly affects the computational cost of the brute-force search for the optimal parameters. On first sight, the initial guesses for $Z 1$ and $Z 2$ seem to be much less accurate (the fit in step one produced a deviation of approximately $175 \mathrm{~nm}$ for the example shown) than for $D$. However, this is not strictly correct. Since the acquired data are interferometric, appropriate fits are periodic, finding values within \pm one fringe order. We therefore recommend choosing the parameter range as $\pm 1 \%$ from the initial guesses (see step 1) and $\pm 1 \%$ from the two adjacent fringe orders.

In case an experiment involving a confined liquid is performed, the parameter space can be further reduced as follows: The distance of closest approach during the experimental runs is used for a pseudo-contact calibration assuming the gap thickness to be zero. Fitting the measured fringes with this assumptions results in an approximation for $Z 1+Z 2$. This approximation is used as a constraint for $Z 1+Z 2$. This sum has to be within a certain range of the pseudo-contact position. The range must be large enough to ensure that it contains the real contact position. In most cases, a range of $+1 \mathrm{~nm}$ (to account for noise in the pseudo-contact position) and $-50 \mathrm{~nm}$ is sufficient to ensure finding the real contact position by performing the aforementioned final calibration.

Figure 4(a) shows the calculated primary fringe profile (blue solid line) for the fitted mica thicknesses $Z 1$ and $Z 2$ and the fitted gap thickness $D$ in comparison with the measured fringe pattern (image at the top) and the extracted fringe positions (green dashed lines). The spectrum calculated on the basis of the calculated layer thicknesses shows excellent agreement with the experimental data. The fitted mica thicknesses $Z 1$ and $Z 2$ also allow calculating a contact position. Figure 4(b) shows the calculated fringe profile for contact in comparison with the actual contact fringe positions. This fit also shows excellent agreement. At this point, we want to stress that the measured contact position was only used to illustrate the accuracy of the demonstrated method but not for the evaluation.

For the data shown here, the calculated fringe positions are slightly red shifted by $0.057 \pm 0.017 \mathrm{~nm}$ compared to the measured fringe positions. However, in other comparable experiments, we also measured offsets that were blue shifted. These errors may arise due to the noise from the light source, systematic error that arises from re-focusing the light after injection of the liquid, the presence of an adsorbed layer of ambient gas, or small differences in dispersion between the literature value and real value of both the mica spacer and the silver mirrors.

The approaches presented here for reducing the parameter space allow for this analysis to be done in a single day on a desktop computer. Without the minimization of the parameter space, we estimate a computation time of multiple weeks.

\section{Step 3: Using the spacer thicknesses to analyze experimental data}

The last step of the method is equivalent to a conventional analysis with multilayer matrix method to extract gap thicknesses as described elsewhere. ${ }^{11}$

To demonstrate the applicability of our data analysis method, we recorded a force profile of $\left[\mathrm{C}_{2} \mathrm{C}_{1} \mathrm{Im}\right]\left[\mathrm{NTf}_{2}\right]$ confined between two mica sheets of different thicknesses in an independent experiment. Figure 4(c) shows the measured force profile at small separations, analyzed according to the procedure described here and without reference to a contact calibration. It clearly shows four distinguishable layers 
A

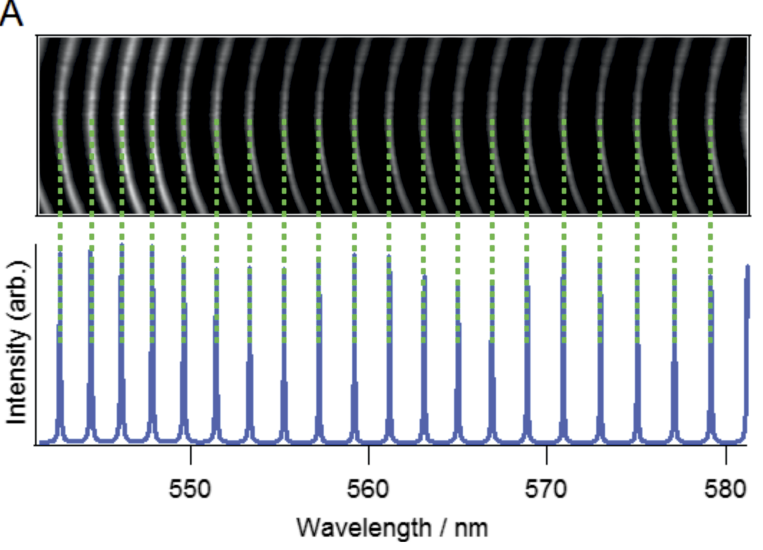

B
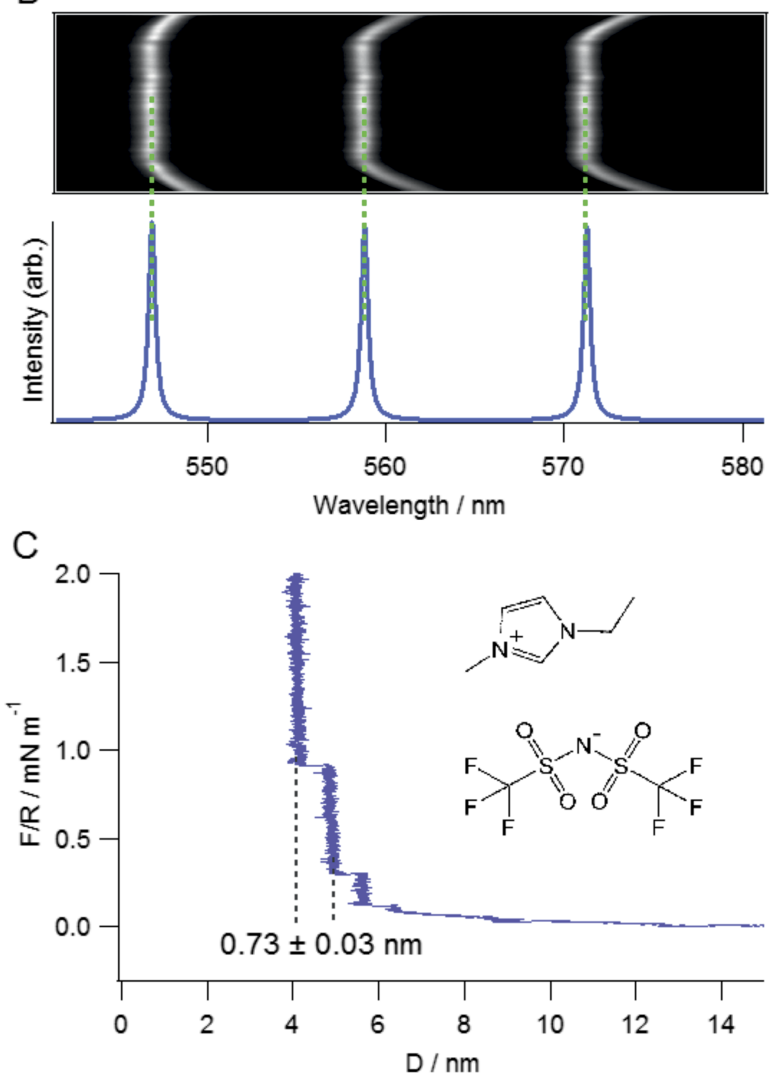

FIG. 4. Results of the fitting procedure and force profile of $\left[\mathrm{C}_{2} \mathrm{C}_{1} \operatorname{Im}\right]\left[\mathrm{NTf}_{2}\right]$ between mica sheets. (a) Experimental interferogram showing primary FECO fringes obtained at a moderate distance between the surfaces. The spectrum underneath corresponds to the fit. The dashed lines represent the fringe positions extracted from the measured FECO fringes. (b) Comparison between the experimentally determined contact position (not used to obtain the fit) and the calculated contact spectrum obtained through the fit in (a). (c) Force profile of the structural forces arising from the layering of dry $\left[\mathrm{C}_{2} \mathrm{C}_{1} \mathrm{Im}\right]\left[\mathrm{NTf}_{2}\right]$ anion/cation layers between mica surfaces.

with a thickness of $0.73 \pm 0.03 \mathrm{~nm}$. The thicknesses of the four observed layers are in excellent agreement with other studies. ${ }^{21-25}$ Further, although a direct comparison between force magnitudes is not strictly informative due to the strong effect of small amounts of water on the interfacial structure, ${ }^{25}$ we find that both the layer thicknesses and the magnitude of the forces measured agree well with other SFB measurements. ${ }^{24,25}$ This demonstrates the applicability of the presented method for measuring absolute distances without contact calibration.
Although performing a contact calibration in this case does not cause surface damage, the test experiment acts as proof-of-principle and demonstration that the procedure may be applied in many instances where contact is not possible or desirable, e.g., with the graphene-SFB.

\section{SUMMARY}

In summary, we have presented a method for determining the thickness of the spacer layers inside a surface force balance/apparatus without performing a contact calibration. To the best of our knowledge, we are the first to report such a method. Notably, our method provides a means to carry out the analysis of the spacer layer thickness on a desktop PC within a day rather than weeks.

We have measured the interlayer distance, surface separation, and associated surface forces of dry $\left[\mathrm{C}_{2} \mathrm{C}_{1} \operatorname{Im}\right]\left[\mathrm{NTf}_{2}\right]$ across two mica surfaces of unequal thickness. We find excellent agreement with values measured earlier by our group and other reports in the literature, which showcases the strength and accuracy of our approach.

The contact-free calibration method is amenable to both symmetric and asymmetric interferometers, opening up the possibility to perform experiments on sensitive surfaces or surfaces that require preparation outside of the apparatus, such as the single layer graphene surfaces mounted on (asymmetric) polymer spacer layers as recently reported in our laboratory. ${ }^{14,26}$

\section{SUPPLEMENTARY MATERIAL}

See supplementary material for a comparison of methods for summing secondary fringe intensities and the effect of mismatch between the refractive indexes of the glue layer and underlying quartz lens.

\section{ACKNOWLEDGMENTS}

The work was supported by the European Research Council (No. ERC-2015-StG-676861), the Leverhulme Trust (No. RPG-2015-328), and the John Fell Fund (Oxford). The project has received funding from the European Union's Horizon 2020 research and innovation programme under Grant Agreement No. 674979-NANOTRANS. C.D.v.E. acknowledges financial support from Zvi and Ofra Meitar Magdalen Graduate Scholarship. We gratefully acknowledge S. Coles for assistance.

${ }^{1}$ J. N. Israelachvili and D. Tabor, Proc. R. Soc. A 331, 19 (1972).

${ }^{2}$ R. G. Horn and J. N. Israelachvili, J. Chem. Phys. 75, 1400 (1981).

${ }^{3}$ J. N. Israelachvili and G. E. Adams, Nature 262, 774 (1976).

${ }^{4}$ J. Fréchette and T. Kyle Vanderlick, Langmuir 17, 7620 (2001).

${ }^{5}$ A. M. Smith, A. A. Lee, and S. Perkin, J. Phys. Chem. Lett. 7, 2157 (2016).

${ }^{6}$ R. M. Pashley, J. Colloid Interface Sci. 80, 153 (1981).

${ }^{7}$ S. Perkin, R. Goldberg, L. Chai, N. Kampf, and J. Klein, Faraday Discuss. 141, 399 (2009).

${ }^{8}$ J. Israelachvili, J. Colloid Interface Sci. 44, 259 (1973).

${ }^{9}$ M. Born and E. Wolf, Principles of Optics, 4th ed. (Pergamon Press, 1970).

${ }^{10}$ J. M. Levins and T. K. Vanderlick, Langmuir 10, 2389 (1994).

${ }^{11}$ M. Heuberger, Rev. Sci. Instrum. 72, 1700 (2001).

${ }^{12}$ M. T. Clarkson, J. Phys. D: Appl. Phys. 22, 475 (1989). 
${ }^{13}$ D. F. Kienle and T. L. Kuhl, Anal. Chim. Acta 936, 236 (2016).

${ }^{14}$ C. D. Van Engers, N. Cousens, V. Babenko, J. Britton, B. Zappone, N. Grobert, and S. Perkin, Nano Lett. 17, 3815 (2017).

${ }^{15}$ M. Heuberger, G. Luengo, and J. Israelachvili, Langmuir 13, 3839 (1997).

${ }^{16}$ J. Klein and E. Kumacheva, J. Chem. Phys. 108, 6996 (1998).

${ }^{17}$ S. Perkin, Phys. Chem. Chem. Phys. 14, 5052 (2012).

${ }^{18}$ K. M. McPeak, S. V. Jayanti, S. J. P. Kress, S. Meyer, S. Iotti, A. Rossinelli, and D. J. Norris, ACS Photonics 2, 326 (2015).

${ }^{19}$ D. F. Kienle, J. V. De Souza, E. B. Watkins, and T. L. Kuhl, Anal. Bioanal. Chem. 406, 4725 (2014).

${ }^{20}$ C. Sanderson and R. Curtin, J. Open Source Software 1, 26 (2016).

${ }^{21}$ R. Atkin, S. Z. El Abedin, R. Hayes, L. H. S. Gasparotto, N. Borisenko, and F. Endres, J. Phys. Chem. C 113, 13266 (2009).

${ }^{22}$ R. Hayes, S. Z. El Abedin, and R. Atkin, J. Phys. Chem. B 113, 7049 (2009).
${ }^{23}$ H.-W. Cheng, P. Stock, B. Moeremans, T. Baimpos, X. Banquy, F. U. Renner, and M. Valtiner, Adv. Mater. Interfaces 2, 1500159 (2015).

${ }^{24}$ A. M. Smith and S. Perkin, J. Phys. Chem. Lett. 6, 4857 (2015).

${ }^{25}$ H.-W. Cheng, J.-N. Dienemann, P. Stock, C. Merola, Y.-J. Chen, and M. Valtiner, Sci. Rep. 6, 30058 (2016).

${ }^{26}$ J. Britton, N. E. A. Cousens, S. W. Coles, C. D. Van Engers, V. Babenko, A. T. Murdock, A. Koo, S. Perkin, and N. Grobert, Langmuir 30, 11485 (2014).

${ }^{27}$ We note that performing a contact calibration by squeezing the surfaces together at the end of an experiment, at which point surface damage might not be an issue, is not usually possible due to strongly adsorbed layers of the liquid or added medium of interest which prevent direct contact between the solid surfaces and cannot be squeezed out under the pressures accessible in the apparatus. 